\title{
Switched Predictive Control Design for Optimal Wet-clutch Engagement
}

\author{
Abhishek Dutta* Clara M. Ionescu* Bart Wyns* \\ Robin De Keyser* Julian Stoev ${ }^{* *}$ Gregory Pinte ${ }^{* *}$ \\ Wim Symens ${ }^{* *}$ \\ * Dept. of Electrical Energy, Systems and Automation, Ghent \\ University, Technologiepark 913, 9052 Zwijnaarde, Belgium. (e-mail: \\ dutta.abhishek@ugent.be). \\ ** Flanders' Mechatronics Technology Center, Celestijnenlaan 300D, \\ 3001 Heverlee, Belgium.
}

\begin{abstract}
Identification and control of wet-clutches has been recognized as a challenging control problem, due to their non-linear time-varying dynamics and their discontinuous switching between filling and slipping phases. In this paper, our approach is based on a full (non)linear system identification of the filling and slipping phases, followed by two closed loop model predictive controllers (MPC) for the two phases and switching between these controllers at suitable moments for optimal torque transfer. Further, it is shown that an intelligent formulation of the switching function is beneficial to the robustness against time-varying model parameters. Finally, our control technique is validated on a real-life wet-clutch test-bench to obtain optimal engagement.
\end{abstract}

Keywords: automotive control, predictive control, reference adaptive control, hybrid systems

\section{INTRODUCTION}

The wet-clutch is a complex mechanical device that transmits torque from its input axis to its output by means of friction, often used in power transmissions of agricultural machines and off-road vehicles to selectively engage gear elements (G.Pinte et al. (2011b)). These machines are operated through several years and under varying environmental conditions such that clutches undergo significant amount of wear and tear. As a result, the dynamics change and the control signals which lead to an optimal engagement need to be adapted during the transmission's life cycle. Hence, robust control of wet clutches has been recognized as a necessary and highly challenging industrial control problem (Z.Sun and K.Hebbale (2005)).

The schematic of a typical wet-clutch is given in Figure 1. The input axis of the clutch is connected to a drum with a first set of friction plates (clutch plates), while a second set of friction plates (clutch discs) are connected to the output axis. An electro-hydraulic pressure-regulated proportional valve regulates the pressure inside the clutch against a return-spring to keep the piston away from plates when not actuated. The control objective is to achieve a fast engagement with minimum output torque peaks for the clutch, ensuring driver comfort.

Modeling and control of dry clutches has received considerable attention in research, only considering a stick-

\footnotetext{
* A. Dutta and Y. Zhong are supported within the framework of the LeCoPro project (grant nr. 80032) of the Institute for the Promotion of Innovation through Science and Technology in Flanders (IWTVlaanderen). C.M. Ionescu is financially supported by the Flanders Research Center (FWO).
}

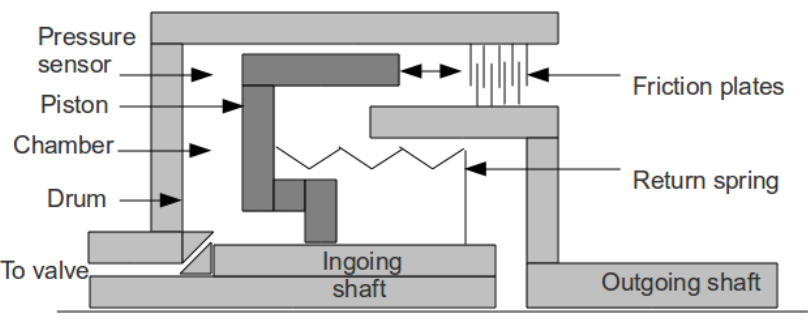

Fig. 1. Wet clutch design

slip hybrid model for analysis. A slip control using linear quadratic regulator (LQR) with force on clutch piston as input is developed in (Glielmo and Vasca (2000)). Explicit MPC is used by (Heijden et al. (2007)) with a linear cost function and parametric (suboptimal) MPC by (Amari et al. (2009)) for slip control in dry clutches, and (Dolcini et al. (2010)) has concluded that an online MPC scheme for clutch control is not practically implementable due to high computation costs.

Over the last three decades Model Predictive Control (MPC) has occupied the center stage in the control research community and had a tremendous impact on the process industry as well (H.Lee (2011)). Through the past decade there is a gradual shift to mechatronic domain, mainly fueled by progress in 'fast MPC' algorithms. Recently, (A.Dutta et al. (2011)) and (A.Dutta et al. (2012)) have shown that good engagement can be achieved on wet clutches by designing robust MPC for pressure tracking by using only a linear fill model. A two-level Iterative Learning Control (ILC) has been used by (G.Pinte et al. (2011a)) with models for both fill and slip phases. These 
schemes use some form of offline learning of the unknown references for optimal engagement.

In this paper, we propose to formulate the reference trajectories for filling and slipping online and track them with two disjoint linear MPC controllers. The design of an on-line switching function between the two MPCs will be shown to provide additional robustness against implicit time-varying dynamics.

The paper is organized as follows. Section 2 presents insights into the physical model of a wet-clutch and motivates the control approach. Section 3 gives an overview of the identification algorithm and the identified model parameters. Section 4 describes the proposed 'Switched MPC' design framework with simulation results. Section 5 evaluates our control performance with respect to the quality of the wet-clutch engagement. Section 6 summarizes the main outcome of this paper and suggests next steps. The work presented in this paper is part of ongoing research within the Learning Control for Production Machines (LeCoPro) project ${ }^{1}$.

\section{MATHEMATICAL CLUTCH MODEL}

In general, such a complex system can be decomposed into the clutch itself and the source of actuation, i.e. a hydraulic mechanism. The control process can be further divided into three phases: Filling, Slipping and Engagement. The Filling phase refers to the build up of the pressure in the clutch until the first contact of the piston with the friction plates takes place. In general, it is considered that there is no torque transferred in this phase. Next, the physical contact triggers the slipping phase where the torque loss due to kinetic friction(i.e. deviations from steady state torque output) is causing a rotational speed difference between the input-output axes. As soon as these speeds are the same, the clutch is in the engagement phase with sufficient static friction. However, in the real wet-clutch setup, there is a change in rotational speeds just before the physical contact, an effect referred to as drag.

The overall block diagram of a 'Wet-clutch' to highlight the flow of physical variables is illustrated in Figure 2. The 'Current' is the input to the 'Hydraulics' subsystem which drives a 'Clutch' subsystem. The 'Engine Torque' can be treated as a known disturbance to the 'Clutch'. The measured sensory outputs are 'Engine and Vehicle speeds, Torque outputs' and 'Pressure' from 'Hydraulics'.

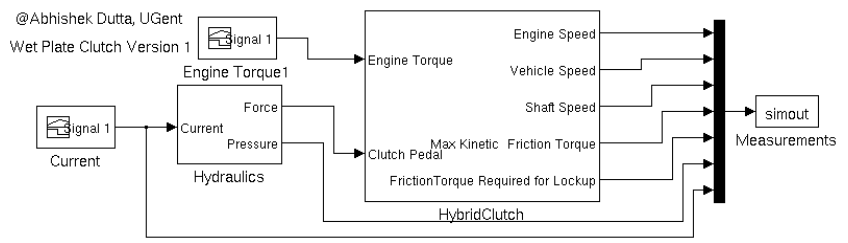

Fig. 2. Input-output schematic of wet clutch subsystems

\subsection{Hydraulics}

This system can be split into three parts: the valve, the hydrodynamics and the mechanical subsystems

\footnotetext{
1 www.lecopro.org
}

(M.J.W.H.Edelaar (1996)). The current enters the system through a solenoid driven proportional pressure reducing valve, which delivers the required pressure.

For the modelling of the valve part, consider the turbulent flow through an orifice that has the following equation:

$$
|\phi|=\gamma\left(\theta_{\text {oil }}\right) A_{\text {orifice }}\left(\left|x_{\text {stem }}\right|\right) \sqrt{\left|p_{\text {clutch }}-p_{\text {pump } / \text { tank }}\right|}
$$

where $\gamma\left(\theta_{\text {oil }}\right)$ depends on the oil properties and temperature. The clutch, pump and tank pressures are $p_{\text {clutch }}, p_{\text {pump }}, p_{\text {tank }}$ respectively. The orifice area $A_{\text {orifice }}$ is a function of the stem position $x_{\text {stem }}$.

The steady state situation of the valve yields the current $(I)$ :

$$
I \propto p_{\text {clutch }} A_{\text {stem }}+k_{\text {stem }} x_{\text {stem }}
$$

where $A_{\text {stem }}, k_{\text {stem }}$ are the area, stiffness of the valve stem respectively.

The second part, the hydrodynamics, describe the relation between oil flow and oil pressure. From the mass conservation principle applied to the filling phase, we have that:

$$
\dot{p}_{\text {clutch }}(t)=\left[\frac{V_{0}}{k_{s}}+\frac{A_{\text {piston }}^{2}}{k}\right]^{-1} \phi(t)
$$

where $V_{0}$ is the chamber volume, $k_{s}$ the oil compressibility, $k$ the return spring constant and $x_{\text {piston }}, A_{\text {piston }}$ are the piston position and area respectively. For the subsequent slip and engagement phases we have:

$$
\dot{p}_{\text {clutch }}(t)=\frac{k_{s}}{V_{0}+A_{\text {piston }} x_{\text {piston }}(t)} \phi(t)
$$

Finally, the mechanical part gives the normal force of the piston:

$$
F_{n}=p_{c l u t c h} A_{\text {piston }}-k x_{\text {piston }}
$$

\subsection{Clutch}

Ideally, the filling phase has no direct effect on the output slip and torque measurements. Therefore, the slipping phase dynamics can be described by the following equations:

$$
\begin{array}{r}
J_{e} \dot{\omega}_{e}=\tau_{e n}-b_{e} \omega_{e}-\tau_{c l} \\
J_{v} \dot{\omega}_{v}=\tau_{c l}-b_{v} \omega_{v}
\end{array}
$$

where $\tau_{e n}, \tau_{c l}$, are the input engine torque and the torque transmitted through the clutch, $J_{e}, J_{v}$ are the moments of inertia, $b_{e}, b_{v}$ damping rates and $\omega_{e}, \omega_{v}$ angular velocities for engine and transmission, respectively.

A Coulomb friction model can be used to relate the input normal force $F_{n}$ supplied by Hydraulics, to the clutch variables (Dassen (2003)):

$$
\tau_{c l}=\operatorname{sign}\left(\omega_{e}-\omega_{v}\right) \cdot \frac{2}{3} R F_{n} \mu_{k}
$$

with the torque capacity as a function of its size $R$ and kinetic friction characteristic $\mu_{k}$. Notice that $\mu_{k}$ varies with the angular velocities and the contact time with the friction plates. 
Finally, the clutch is engaged when the following condition is fulfilled:

$$
\text { Slip }=\omega_{e}-\omega_{v}=0
$$

\subsection{Hypothesis}

Based on the above physical interpretation, the following conclusions can be drawn. The 'Wet-Clutch' can be broken down into two subsystems: 'Hydraulics' and 'Clutch' and each subsystem into three phases: 'Fill', 'Slip' and 'Engagement'. The dynamics of the 'Hydraulics' subsystem evolve in all the three phases, but the dynamics of the 'Clutch' subsystem are visible only in the latter two phases. Thus, the behaviour of the clutch can be uniquely described by a 'subsystem-phase' combination which we henceforth denote as a 'region'. All these regions are non-linear and the Fill-phase has additional temperature dependence through (1).

The Fill-phase in the Hydraulics subsystem, i.e. 'HydraulicsFill' region, is where the dynamics start by filling the chamber of the clutch, with a current input building up output pressure on the piston. The region 'Wet-clutchSlip' denotes the Slip phase over the Wet-clutch system i.e. a model from input current to output slip by combining the equations presented in Hydraulics and Clutch subsections. If a (non)linear system identification of 'Wet-clutchSlip' is performed together with 'Hydraulics-Fill', the full potential of these models can be exploited by designing a switched MPC, which will be presented in section 4 . By means of on-line switching, this scheme tackles the temperature variance.

The Engagement phase is not critical for control because by this stage, the fast filling is ensured by Fill control and low output torque is ensured by Slip control. The objective of the Engagement phase is to keep the Wetclutch engaged, and can easily be achieved by a feedforward step current, and will not be considered in the control strategies in the remainder of this paper.

\section{SYSTEM IDENTIFICATION}

For performing linear and non-linear estimation on the real clutch setup for the 'Hydraulics-Fill' and 'Wet-clutch-Slip' regions, the following steps are followed:

(1) Design the input covering the bandwidth of interest.

(2) Estimate the frequency response of the system and identify a parametric model of the frequency response, either state space or transfer function model.

(3) Estimate a polynomial non-linear state space model. The linear model parameters are used as an initial guess for the non-linear least squares estimation of the model parameters.

(4) Validate the estimated model with a new input.

\section{1 'Hydraulics-Fill' models}

Transfer function linear model (I to P): The dynamics between the current $I$ to the valve and the generated oil pressure $P$ in the chamber of the clutch, while the piston is located between the completely open clutch position and the friction plates, is given by:

$$
\frac{P-1}{I-0.04}=\frac{0.234 q^{-2}}{1-2.736 q^{-1}+2.515 q^{-2}-0.7764 q^{-3}}
$$

where $q^{-1}$ is the backward shift operator and sampling time is 1ms. Polynomial Non-Linear State Space (PNLSS) model (I to P) is given by (W.D.Widanage et al. (2011)):

$$
\begin{aligned}
x(t+1) & =A x(t)+B u(t)+E v(t) \\
y(t) & =C x(t)+D u(t)+F v(t)
\end{aligned}
$$

with $A \in \mathbb{R}^{3 \times 3}, B \in \mathbb{R}^{3 \times 1}, C \in \mathbb{R}^{1 \times 3}, D \in \mathbb{R}, E \in \mathbb{R}^{3 \times 8}$ and $F \in \mathbb{R}^{1 \times 8}$. The vector $v(t)$ contains the state and input monomials and is $v(t)=\left[x_{1}^{2}, x_{2}^{2}, x_{3}^{2}, u^{2}, x_{1}^{3}, x_{2}^{3}, x_{3}^{3}, u^{3}\right]^{\prime}$.

\section{2 'Wet-clutch-Slip' models}

A linear state space model (I to S) captures the dynamics between the current $I$ to the valve and the slip $S$ due to the change in rotational speeds between the input-output axes. The piston is located in the area within the vicinity of the friction plates and the complete closure of the clutch. The linear model is given by,

$$
\frac{S}{I}=\frac{-0.046+0.088 q^{-1}-0.054 q^{-2}-0.013 q^{-3}}{1-3.96 q^{-1}+5.9 q^{-2}-3.9 q^{-3}+0.96 q^{-4}}
$$

The polynomial non-linear state space model (I to $\mathrm{S}$ ) is given by:

$$
\begin{aligned}
x(t+1) & =A x(t)+B u(t)+E v(t) \\
y(t) & =C x(t)+D u(t)+F v(t)
\end{aligned}
$$

with $A \in \mathbb{R}^{4 \times 4}, B \in \mathbb{R}^{4 \times 1}, C \in \mathbb{R}^{1 \times 4}, D \in \mathbb{R}$, $E \in \mathbb{R}^{4 \times 15}$ and $F \in \mathbb{R}^{1 \times 15}$. The vector $v(t)$ contains the state and input monomials and is $v(t)=$ $\left[x_{1}^{2}, x_{2}^{2}, x_{3}^{2}, x_{4}^{2}, u^{2}, x_{1}^{3}, x_{2}^{3}, x_{3}^{3}, x_{4}^{3}, u^{3}, x_{1}^{4}, x_{2}^{4}, x_{3}^{4}, x_{4}^{4}, u^{4}\right]^{\prime}$.

Notice that only the structure of the obtained non-linear models is presented. The exact values of the coefficients are superfluous and are concealed as per the project agreement.

\section{THE SWITCHED MPC-EPSAC APPROACH}

MPC is a form of control in which the current control action is obtained by solving on-line, at each sampling instant, a finite horizon open-loop optimal control problem, using the current state of the plant as the initial state. The optimization yields an optimal control sequence and the first control in this sequence is applied to the plant (i.e. the receding horizon concept). The MPC concept is illustrated in Figure 3. In particular, the efficient implementation of the MPC-EPSAC (Extended Prediction Self Adaptive Control) approach based on filtering technique makes it a suitable choice for real-time mechatronic applications.

\section{$4.1 E P S A C-M P C$}

The process is modeled as in (R.DeKeyser (2003)):

$$
y(t)=x(t)+n(t)
$$

with $y(t), x(t), n(t)$ as process output, model output, process/model disturbance respectively. The fundamental 


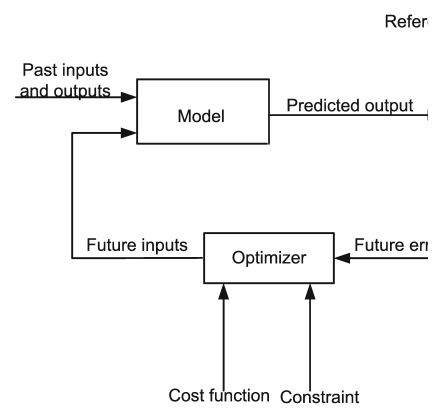

Fig. 3. Schematic of MPC

step is based on the prediction over the basic process model given by:

$$
y(t+k \mid t)=x(t+k \mid t)+n(t+k \mid t)
$$

where $y(t+k \mid t)$ is the prediction of process output at time instant $t$, over prediction horizon $N_{2}$, based on prior measurements and postulated values of inputs. Prediction of model output $x(. \mid$.) and of colored noise process $n(. \mid)$. can be obtained by the recursion of process model and filtering techniques respectively. The colored noise can be modeled as:

$$
n(t)=\frac{C\left(q^{-1}\right)}{D\left(q^{-1}\right)} e(t)
$$

with $e(t)$ as white noise, designed for robustness against plant-model mismatch. The optimal control is then obtained by minimizing the following cost function:

$$
\begin{gathered}
V(U)=\Sigma_{k=N_{1}}^{N_{2}}[r(t+k \mid t)-y(t+k \mid t)]^{2} \\
+\lambda \Sigma_{k=0}^{N_{u}-1}[\Delta u(t+k \mid t)]^{2}
\end{gathered}
$$

with $\lambda$ being the control penalty and $r(. \mid$.$) the desired$ reference trajectory (i.e. low pass filter). $N_{1}$ is the process dead time and the future control increments $\Delta u(. \mid$.$) are$ allowed to vary over the prediction horizon $N_{2}$. The solution with constraints on inputs and/or outputs, if feasible, is obtained through quadratic programming. Only the first optimal control input is applied to the plant.

\subsection{Switched MPC Design}

Hybrid dynamical systems are a combination of continuous dynamics with discontinuous switching. Such systems can be approximated arbitrarily well as piecewise affine systems. In mechatronic systems, when making a transition from a (non)linear model to another, the associated inputs or outputs may change as well. This forces a change in the order/meaning of the associated models/states as well. These systems can be categorized as a specific type of hybrid dynamical systems, which we introduce here as 'Switched dynamical systems'.

The complete dynamic behavior of the 'Wet-clutch', if identified successfully, represents a 'Switched dynamical system'. This is due to the fact that its region of operation jumps from 'Hydraulics-Fill' (input current, I to output pressure, P) to 'Wet-clutch-Slip' (input current, I to output slip, S), which are then two completely different models with different orders and state representations. To control such 'Switched dynamical systems', a 'Switched

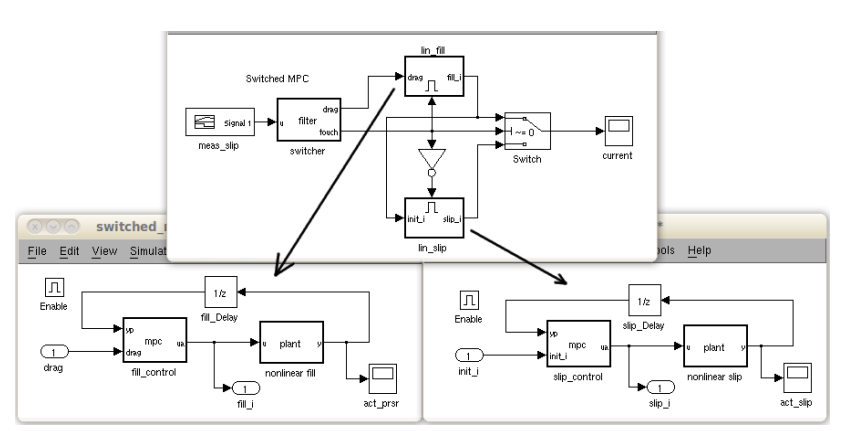

Fig. 4. Switched MPC for switched dynamical systems

MPC' scheme must be designed with as many MPCs as the number of regions. The switching moment is triggered by observing certain changes in the state variables. Notice that, the switching time may vary with varying conditions. This implies that the 'Switched MPC' scheme is intrinsically robust.

Proposition 1. Design of an on-line 'Switching function' increases robustness of the closed loop regulation of a timevarying switching system.

To validate the above, consider the wet-clutch control objective of fast and smooth engagement. It translates to the following:

(1) Fast filling: follow a high pressure reference in the 'Hydraulics-Fill' until there is a first drop in slip (i.e. drag point).

(2) Prepare for smooth engagement: switch to a low pressure reference also in 'Hydraulics-Fill' until a second drop appears in slip (i.e. contact with the friction plates).

(3) Smooth engagement: switch to the 'Wet-clutch-Slip' and track a sigmoid reference ensuring that the maximum jerk is bounded.

In conventional control, one a priori specifies these reference trajectories; although they depend on the oil temperature which varies during operation, i.e. (1). The proposed methodology is robust to these temperature changes: eg. in the case of high temperature, the contact and drag points would appear earlier, leading to an earlier entry to Slip control. Therefore, the on-line switching leads to adaptive references which provide additional robustness of the closed loop.

To generalize, consider a 'Switched dynamical system' composed of a series of non-linear regions connected by discontinuous jumps. The switched MPC formulation is based on the following steps:

(1) Identify linear and non-linear models for each of the continuous regions.

(2) Design stand-alone MPC controllers for each region, separately.

(3) Use the linear model for MPC predictions and the non-linear model as the simulated plant. Tune the various control parameters and disturbance filter for robustness against model-plant mismatch.

(4) Implement an appropriate switching function for adapting references on-line to ensure robustness against time-variance (aka Proposition 1). 


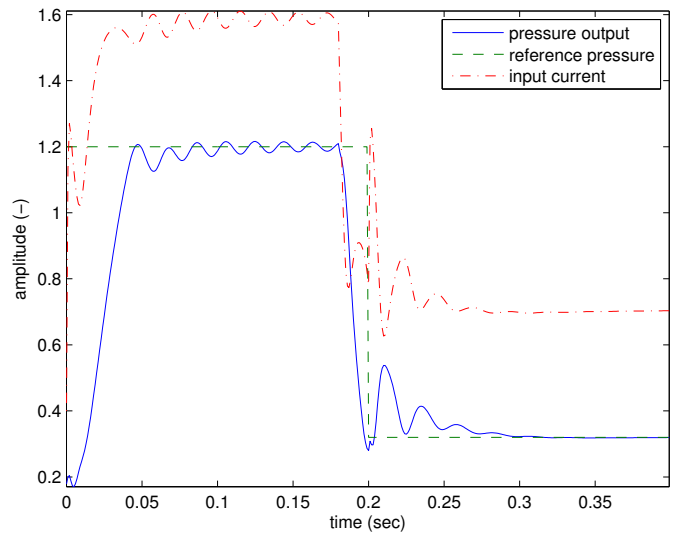

Fig. 5. $M P C_{\text {fill }}$ regulation performance

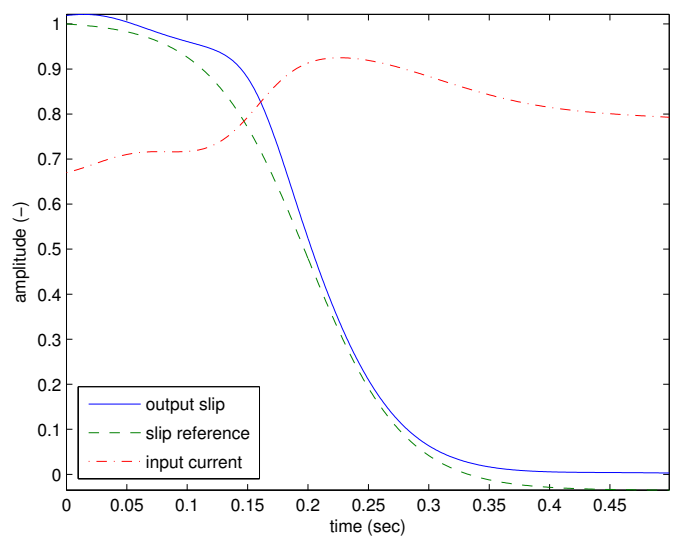

Fig. 6. $M P C_{\text {slip }}$ tracking performance

(5) Implement the 'Switched MPC' structure on the real machine by replacing the non-linear simulated plant by the actual measurements.

In our case the 'Switched MPC' is applied to the wet-clutch and the corresponding block diagram is shown in Figure 4. It has two continuous regions of operation, namely 'Hydraulics-Fill' and 'Wet-clutch-Slip'. The corresponding linear and non-linear models for the two regions have been given in section 3 .

First, a robust EPSAC-MPC controller, $M P C_{f i l l}$ is designed for 'Hydraulics-Fill' based on the linear model of (10) and simulated non-linear plant of (11). It is tuned for tracking a step-up followed by step-down pressure setpoint. A first-order reference trajectory with time constant $T_{c}$ is used over the set-point (i.e. a low pass filter). The obtained EPSAC-MPC parameters are $\left(N_{1}=1, N_{2}=\right.$ $\left.20, N_{u}=1, \lambda=10^{4}, T_{c}=13\right)$. The default integrator is used as disturbance filter. The simulation results are plotted in Figure 5, showing good performance.

Next, the tuning process is repeated, now for the 'Wetclutch-Slip' based on the linear model of (12) and simulated non-linear plant of (13) for tracking a nominal sigmoid slip reference. The parameters for $M P C_{\text {slip }}$ then are $\left(N_{1}=1, N_{2}=5, N_{u}=1, \lambda=10^{5}, T_{c}=1\right)$, with integrated
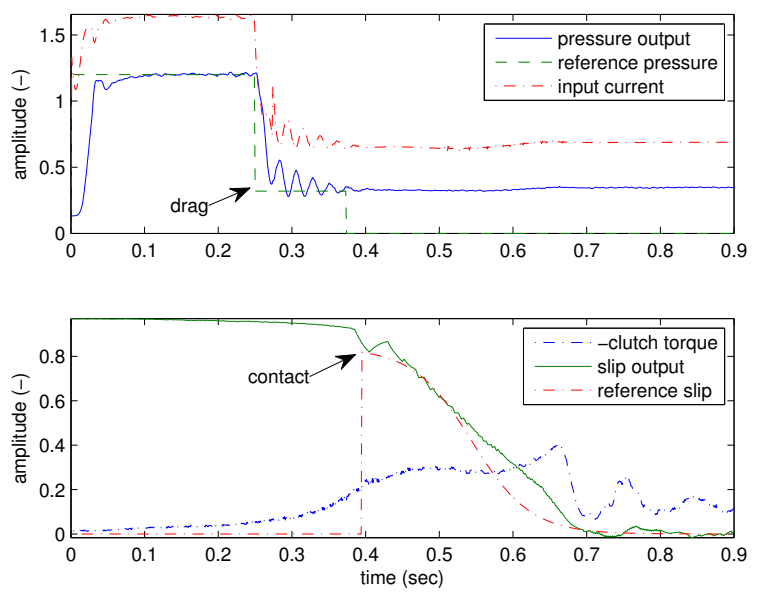

Fig. 7. Switched EPSAC control (top) and engagement quality (bottom)

white noise as disturbance model. The simulation results are plotted in Figure 6, again showing good performance.

\section{EXPERIMENTAL RESULTS}

Since the theoretical developments were satisfactory in simulations, the Robust MPC and Switched MPC are now rigorously tested on the real non-linear hybrid clutch testbench. We define an optimally controlled engagement, as the engagement with output torque bounded by 0.5 and has an engagement time within 1s. The system has the following constraints:

$$
\begin{array}{r}
0 \leq \text { Current }(\text { OverallInput }) \leq 2 \\
0 \leq \text { Pressure }\left({ }^{\prime} \text { Hydraulics }- \text { Fill'Output }\right) \leq 1.4 \\
0 \leq \text { Slip }\left({ }^{\prime} \text { Wet }- \text { clutch }- \text { Slip }{ }^{\prime} \text { Output }\right) \leq 1
\end{array}
$$

The Switched EPSAC-MPC is realized on the real clutch test-bench for optimal engagement as follows:

(1) Design a switching function which monitors the measured slip for a first and second drop, called drag and contact respectively. These are tuned from system knowledge to anywhere between $(94-97) \%$ and $(85-$ $90) \%$ of the initial filtered slip value for drag and contact respectively.

(2) Start with $M P C_{\text {fill }}$ maintaining a high pressure setpoint (near maximum), ensuring fast filling. This is the 'Hydraulics-Fill' region of operation. Use the previously tuned parameters from 4.2 to control the current based on measured pressure.

(3) When drag is detected, a low pressure set-point must be tracked, i.e. to gently push the piston to the friction plates and get-ready for entering the lowbandwidth Slip phase. Notice that for simplicity, we use the same $M P C_{\text {fill }}$ controller here.

(4) When the moment of contact occurs, switch to the $M P C_{\text {slip }}$ closed loop control. The final states of $M P C_{\text {fill }}$ cannot be made the initial states of $M P C_{\text {slip }}$, as we have a different model now, i.e. from current to slip. Hence, hot-start the controller with initial input current transferred from $M P C_{\text {fill }}$ and output slip value from measurement. 
(5) Start $M P C_{\text {slip }}$ based on the 'Wet-clutch-Slip' model and tuned parameters from 4.2, manipulating the same input current but with slip as the controlled output. The slip is following a sigmoid reference of the form: $\frac{\text { gain }}{1+\exp (\text { slope } * \text { t) }}, t \in[-\Delta / 2: T s: \Delta / 2)$. The 'gain' is the measured slip value at the time of switching in order to synchronize the reference online. Given the desired time span $\Delta$ for slip to go to 0 , an optimal 'slope' can be computed.

Note that, a reference free approach in the slip phase using reference governors would lead to identical results. Here, the idea is to manipulate the time constant $T_{c}$ of the MPC reference to exponentially decay to 0 slip. Thus the MPC itself replans the slip trajectory together with tracking it.

Further, the switching logic used ensures stability. The $M P C_{\text {fill }}$ determines the time span of fill control, which is same as the switching time $t_{\text {switch }}$, based on the computation of drag and contact times. The linear slip controller is then started at this pre-computed switching instant $t_{\text {switch }}$, and by design, there is no possibility to go back to the fill control phase anymore, which ensures there is no chattering.

A real-time EPSAC is developed with Hildreth Quadratic Programming (Wang (2009)) and implemented over dSPACE platform to achieve turn around times of less than $1 \mathrm{~ms}$. The real-time platform is used to control the wet-clutch test setup and the corresponding results are plotted in Figure 7. This experiment has been performed at operating conditions around $40^{\circ} \mathrm{C}$ under low external load and low breaking torque, with sampling time of $1 \mathrm{~ms}$.

The current builds up gradually in the Fill phase due to the control penalty in $M P C_{\text {fill }}$ ensuring no overshoot in pressure, though it eventually saturates. The drag point is computed on-line at time instant $0.25 \mathrm{~s}$, which indicated a change in the pressure reference to 0.32 . This period is of particular importance as it leads to the Slip phase, and the switching must occur with low pressure or piston velocity. If this condition is not ensured, one cannot prevent a steep slip decrease and consequent output torque peaks (i.e. jerks). The contact is determined just before $0.4 \mathrm{~s}$, leading to a transition to $M P C_{\text {slip }}$ control loop, which tracks a sigmoid slip reference ensuring a smooth engagement within another $0.5 \mathrm{~s}$, confirmed by low torque peaks.

\section{CONCLUSIONS}

This paper presented a novel switched model based predictive control technique for non-linear time-varying mechatronic systems with switching dynamics. The methodology has been successfully validated by optimal engagement of a wet-clutch test-bench system. The design of the switching mechanism leads to an adaptation of reference trajectories, thus maintaining robust performance.

The extension to 'Switched non-linear MPC' and its application to the wet-clutch, especially designed for optimal slip control is ongoing work.

\section{ACKNOWLEDGEMENTS}

We would like to acknowledge Dhammika Widanage (VU Brussel) and Bruno Depraetere (KU Leuven) for their support with identification and control.

\section{REFERENCES}

A.Dutta, C.M.Ionescu, R.DeKeyser, J.Stoev, G.Pinte, and W.Symens (2012). Robust predictive control design for optimal wet-clutch engagement. In American Control Conference, 4576-4581. Montreal, Canada.

A.Dutta, R.DeKeyser, Y.Zhong, B.Wyns, G.Pinte, and J.Stoev (2011). Robust predictive control of a wetclutch using evolutionary algorithm optimized engagement profile. In 15th International Conference on System Theory, Control and Computing, 1-6. Sinaia, Romania.

Amari, R., Alamir, M., and Tona, P. (2009). Experimental evaluation of a hybrid mpc strategy for vehicle start-up with an automated manual transmission. In European Control Conference. Budapest.

Dassen, M. (2003). Modelling and control of automotive clutch systems. In Report number 2003.73. Eindhoven.

Dolcini, P., de Wit, C.C., and Bechart, H. (2010). Dry clutch control for Automotive Applications. Springer.

Glielmo, L. and Vasca, F. (2000). Engagement control for automotive dry clutch. In American Control Conference, 1016-1017. Chicago, USA.

G.Pinte, B.Depraetere, W.Symens, and J.Swevers (2011a). A two-level iterative learning control scheme for the engagement of wet clutches. Mechatronics, 501-508.

G.Pinte, J.Stoev, W.Symens, A.Dutta, Y.Zhong, B.Wyns, R.DeKeyser, B.Depraetere, J.Swevers, M.Gagliolo, and A.Nowe (2011b). Learning strategies for wet clutch control. In 15th International Conference on System Theory, Control and Computing, 467-474. Sinaia, Romania.

Heijden, A., Serrarens, A., Camlibel, M., and Nijmeijer, H. (2007). Hybrid optimal control of dry clutch engagement. International Journal of Control, 1717-1728.

H.Lee, J. (2011). Model predictive control: Review of the three decades of development. International Journal of Control, Automation, and Systems, 415-424.

M.J.W.H.Edelaar (1996). Modeling of a wet plate clutch in a driveline. In WFW Report 96.071. Eindhoven.

R.DeKeyser (2003). Model based predictive control. Invited chapter in UNESCO EoLSS, Oxford, 6.43.16.1.

Wang, L. (2009). Model predictive control system design and implementation using matlab. Springer-Verlag.

W.D.Widanage, J.Stoev, A.VanMulders, J.Schoukens, and G.Pinte (2011). Nonlinear system-identification of the filling phase of a wet-clutch system. Control Engineering Practice, 1506-1516.

Z.Sun and K.Hebbale (2005). Challenges and opportunities in automotive transmission control. In American Control Conference, 3284-3289. Oregon, USA. 